\section{Chronic Pericardial Effusion}

In middle African countries the following causes have to be borne in mind:

(1) Tuberculous pericardial effusion.

(2) Endomyocardial fibrosis.

(3) Chronic idiopathic pericardial effusion.

(4) Malignant pericarditis.

Tuberculous pericardial effusion presents in a similar way to acute benign pericarditis and its main differential diagnosis. The pericardial fluid may be serous or haemorrhagic.

Air contrast studies of the pericardium may reveal thickening of the pericardium.

Suggestive evidence of tuberculous aetiology is shown by a positive Heaf test and historical or radiological evidence of past tuberculosis.

Direct examination of the fluid will show a high cell-count, consisting mostly of lymphocytes, and a raised protein. Direct examination of the fluid deposit by Ziehl-Neelsen staining sometimes shows acid-fast bacilli. Culture and biological tests usually entail a wait for several weeks for confirmation of the diagnosis.

The treatment programme is one of repeated aspiration to relieve tamponade and antituberculous therapy (streptomycin $1 \mathrm{~g}$. and thiacetazone $100 \mathrm{mg}$. with isoniazid $300 \mathrm{mg}$. in divided doses daily) preferably under clinic conditions for a minimum of three months.

The patient should be reviewed clinically and radiologically for development of pericardial fibrosis and calcification. Signs suggestive of pericardial constriction are those of restricted cardiac filling: persistent jugular venous engorgement, hepatic distension and ascites, and a low cardiac output state-low blood pressure, pulsus paradoxus, a third sound, and sometimes atrial fibrillation.

Surgical relief by pericardiectomy is indicated in the presence of constrictive pericarditis.

Endomyocardial fibrosis.-Reference has been made to this cardiac disorder in an earlier article in this series. ${ }^{1}$

In Uganda endomyocardial fibrosis is associated with a $33 \%$ incidence of chronic pericardial effusion. The age group affected is childhood and adolescence. The clinical features are those of pericardial effusion. Confirmation is by pericardiocentesis.

As with tuberculous pericardial effusion, the fluid is proteinrich and clots readily on removal. It is serous, and examination shows the presence of lymphocytes and polymorphonuclear cells. The culture is negative. In the natural course of the

\section{TODAY'S DRUGS}

With the help of expert contributors we publish below notes on a selection of drugs in current use.

\section{Suppository Treatment of Haemorrhoids}

There are many factors which make it difficult to determine the value of suppositories in the treatment of haemorrhoids. Firstly, spontaneous remission is common. Secondly, haemorrhoids can be demonstrated in most people if the clinician tries hard enough. Thirdly, there is no objective evidence that suppositories can effect a cure.

\section{Diagnosis and Treatment}

As precise a diagnosis as possible must be made and other rectal disease should be excluded by sigmoidoscopy. The illness the pericardial effusion may persist for up to five years. With time it tends to dry, leaving the classical features of endomyocardial fibrosis, which can be differentiated only with difficulty from constrictive pericarditis following "tuberculous" pericardial effusion in such instances.

The absolute diagnosis of endomyocardial fibrosis is obtainable by cineangiocardiography (right or left sided), which will demonstrate flattening of the ventricular apex, and atrioventricular incompetence.

There is no known treatment for endomyocardial fibrosis apart from relief of tamponade as necessary. The disease is usually progressive. Heart failure is intractable and there is only a limited response to digitalis and diuretic agents.

Chronic idiopathic pericardial effusion is known in both temperate and tropical environments. The cause is not clear and may have to do with abnormalities of venous drainage of the pericardium. Patients are known to survive for many years and it is known for female patients to survive pregnancy and labour without ill-effects. The radiological and electrocardiographic signs are those of pericardial effusion. The fluid is serous and may contain large amounts of protein. It is possible that chronic idiopathic pericardial effusion in tropical Africa is a variant of endomyocardial fibrosis.

Malignant pericarditis secondary to a primary carcinoma in the breast, kidney, or thyroid is known to occur in tropical countries. It is usually in the form of a haemorrhagic effusion. Air contrast studies will show thickened parietal pericardium, and special staining malignant cells in the effusion deposit.

In childhood especially, Burkitt's tumour may arise in the pericardium and produce signs of a pericardial effusion or pericardial constriction.

\section{Summary}

Pericarditis is an important cardiac disorder in the tropics. It is frequently infective in origin; tuberculous and pyogenic causes are the usual ones, and they can be readily diagnosed and cured. Other specifically tropical causes of pericarditis include endomyocardial fibrosis and amoebic pericarditis.

Features relating to pericarditis as seen under tropical conditions are discussed with regard to diagnosis and management.

I am grateful to the British Heart Foundation for the support they have given me for cardiological studies in Uganda.

REFERENCE

1 Parry, E. H. O., Brit. med. F., 1966, 2, 1119. symptoms which the patient may attribute to haemorrhoids may in fact be due to other conditions such as fissure, fistula, perianal haematoma, redundant skin tags, or hypertrophied anal papillae. When an acute fissure lies concealed between haemorrhoids it is often not diagnosed. It may heal spontaneously, but local analgesics are helpful in reducing the pain on defaecation and promoting a remission. A perianal haematoma has been described as a "painful five-day self-curing lesion" and is best left alone, unless the lesion is very large and painful, when surgical evacuation is justified. Fistulae, redundant skin tags, and anal papillae need surgical treatment. If the complaint is of bleeding or mucous discharge due to first or second degree haemorrhoids, most surgeons would advise injection of the piles with phenol in oil. If the lesion is third degree then injection or operation would be advised. Patients who refuse both suggestions may reduce or even stop the bleeding if they soften the stool by altering the diet or by 
taking laxatives. Failing this suppository treatment may be tried.

There are several kinds of suppository which have been advocated for the treatment of haemorrhoids. One of the most popular is bismuth subgallate co. B.P.C. Others containing analgesics or hydrocortisone are frequently used, and there are further preparations of more uncertain value.

\section{Bismuth Subgallate Co. Supp. B.P.C.}

$\begin{array}{lllllllr}\text { Bismuth subgallate } & & \ldots & \ldots & \ldots & \ldots & \ldots & 200 \mathrm{mg} \text {. } \\ \begin{array}{l}\text { Resorcinol } \\ \text { Zinc oxide } \ldots\end{array} & \ldots & \ldots & \ldots & \ldots & \ldots & \ldots & 60 \mathrm{mg} . \\ \text { Peru balsam } & \ldots & \ldots & \ldots & \ldots & \ldots & \ldots & 120 \mathrm{mg} . \\ \text { Castor oil } \ldots & \ldots & \ldots & \ldots & \ldots & \ldots & \ldots & 60 \mathrm{mg} . \\ \end{array}$

The active ingredients are made up in some suitable fatty basis such as theobroma oil (cocoa butter) or Witepsol. The basic N.H.S. cost for 12 has been estimated as $8 \mathrm{~d}$. in a hospital pharmacy. Unfortunately this preparation still contains resorcinol and balsam of Peru, both of which are skin sensitizers. It was for this reason that balsam of Peru was withdrawn from tulle gras in recent years.

It would be pertinent to have a controlled clinical trial to determine whether this suppository has any advantage over a suppository composed only of the fatty base. tion.

There are two proprietary preparations of similar composi-

ANusol (William R. Warner and Co. Ltd.).

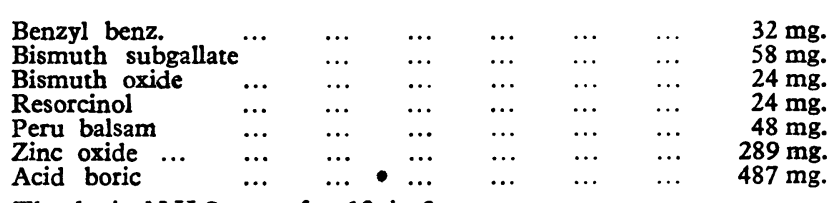

The basic N.H.S. cost for 12 is 2 s.

ANAROIDS (Rybar Laboratories Ltd.).

\begin{tabular}{llllllllr}
\multicolumn{2}{l}{$\begin{array}{l}\text { Bismuth subgallate } \\
\text { Resorcinol }\end{array}$} & $\ldots$ & $\ldots$ & $\ldots$ & $\ldots$ & $\ldots$ & $\ldots$ & $2 \%$ \\
$\begin{array}{l}\text { Acid gallo-tannic } \\
\text { Titanium dioxide }\end{array}$ & $\ldots$ & $\ldots$ & $\ldots$ & $\ldots$ & $\ldots$ & $\ldots$ & $\ldots$ & $1 \%$ \\
$\begin{array}{l}\text { Zinc oxide } \\
\text { Acid boric }\end{array}$ & $\ldots$ & $\ldots$ & $\ldots$ & $\ldots$ & $\ldots$ & $\ldots$ & $\ldots$ & $1 \%$ \\
$\begin{array}{l}\text { Peru balsam } \\
\text { Kaolin }\end{array}$ & $\ldots$ & $\ldots$ & $\ldots$ & $\ldots$ & $\ldots$ & $\ldots$ & $\ldots$ & $2 \%$ \\
Ka & $\ldots$ & $\ldots$ & $\ldots$ & $\ldots$ & $\ldots$ & $\ldots$ & $\ldots$ & $10 \%$ \\
\end{tabular}

The basic N.H.S. cost for 12 is $2 \mathrm{~s}$.

\section{Analgesic Compounds}

When pain is the main complaint in a patient with haemorrhoids, a fissure, a perianal haematoma, or strangulation of the haemorrhoids is probably the cause. Application of a suitable lotion and the use of local analgesics are often a great comfort. As sensitization to these analgesics is not uncommon, they should be used for only a week or two at a time. With this proviso there are two suppositories which can be recommended:

\section{ANUGESIC (William R. Warner and Co. Ltd.).}

Each suppository contains the Anusol ingredients, together with the local analgesic pramoxine $0.9 \%$.

The basic N.H.S. cost for 12 is $3 \mathrm{~s} .8 \mathrm{~d}$.
LOCAN (Duncan, Flockhart and Evans Ltd.).

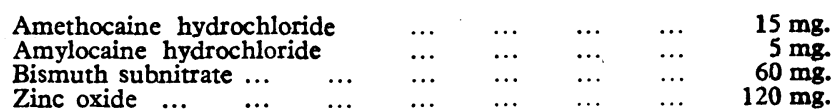

The basic N.H.S. cost for 12 is 3 s.

\section{Hydrocortisone Compounds}

Many of the suppositories recommended for haemorrhoids contain hydrocortisone. Though there is a theoretical objection to the use of hydrocortisone in potentially infected situations, such as a fissure or strangulated haemorrhoids, this is probably unimportant. The real objection to the addition of hydrocortisone is the increase in cost of three- or fourfold. It has been suggested that its beneficial effect, if any, is of damping down the irritation produced by some of the other ingredients of the suppositories such as the analgesics, balsam of Peru, or resorcinol. It is doubtful whether any hydrocortisone-containing suppository is of real benefit in haemorrhoids and therefore none can be recommended.

\section{Other Preparations}

Alcos-Anal (Camden Chemical Co. Ltd.).

$$
\begin{aligned}
& \text { Sodium' salts of purified unsaturated fatty acids derived } \\
& \text { from cod-liver oil } \quad \begin{array}{lllllll} 
& \ldots & \ldots & \ldots & \ldots & \ldots & 11.0 \%
\end{array}
\end{aligned}
$$

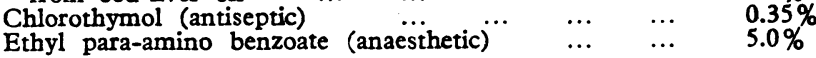$$
\text { The basic N.H.S. cost for } 12 \text { is } 2 \mathrm{~s} \text {. }
$$

There have been many claims that Alcos-Anal suppositories will stop haemorrhoids bleeding and reduce their size. These suppositories contain sodium salts of purified unsaturated fatty acids, which are said to produce an aseptic inflammation. Their main disadvantage is a stinging sensation which persists for ten minutes after the suppository has been inserted into the anus. Polypharmacy has little to commend it and as yet there is no evidence that these preparations cure haemorrhoids.

Biosone (Berk Pharmaceuticals Ltd.).

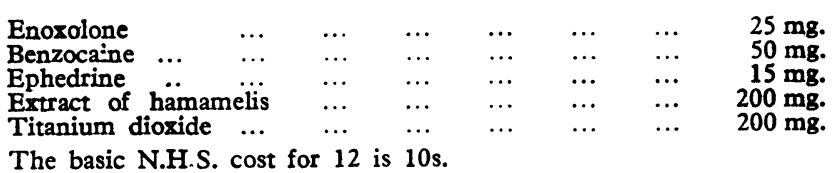

Enoxolone is prepared from a glycoside found in liquorice and has some resemblance to the structure of cortisone. It is anti-inflammatory. Since its value is unproved, the cost of these suppositories is prohibitive.

As there have been no controlled clinical trials in this branch of proctology it is not possible to be dogmatic about any preparation. What is required is a "double blind" trial comparing the effect of some of the above-mentioned preparations with suppositories composed only of a fatty base. For the moment it would appear reasonable to prescribe the analgesic suppositories or bismuth subgallate co. for a short period to give symptomatic relief during an uncomfortable episode. 